\title{
EFFECT OF UNCOMPLICATED PHACOEMULSIFICATION ON INTRAOCULAR PRESSURE
}

\author{
By
El Sayed Mostafa Elewa, Hassan Metwally Bayoumy and Atef Samir Hamed Sherif

Ophthalmology Department, Faculty of Medicine, Al-Azhar University

\begin{abstract}
Background: Cataract and glaucoma are the first and second leading causes of blindness worldwide. Although usually not severe enough to cause blindness, it is not surprising that these two diseases occur simultaneously in many patients. Many studies have demonstrated intraocular pressure reduction after cataract surgery. However, most recent data indicates that intraocular pressure (IOP) reduction after cataract surgery is more significant and sustained than previously thought.

Objectives: The aim of the work is to study and measure the effect of uncomplicated phacoemulsification on intraocular pressure using air puff machine and applanation tonometer at Alazhar University Hospitals.

Patients and Methods: This was a prospective randomized study carried out on 40 eyes who underwent measuring IOP by air puff and applanation tonometer before phacoemulsification and IOL implantation, and one day, one month, three months post-operatively at Al Azhar university hospitals. An informed and written consent was taken from all the patients included in this study.

Results: Our study was carried out on 40 eyes who underwent measuring IOP by air puff and applanation tonometer one-day pre phaco, then underwent phacoemulsification and IOL implantation of the same IOL type, and then measuring of IOP 1 day, 1 week, 1 month and 3 months post-operatively. A decrease in IOP after cataract surgery has been reported. Long term studies have shown a drop in IOP of about $4 \mathrm{~mm} \mathrm{Hg}$ in primary open angle glaucoma (POAG) patients, and non-glaucomatous patients.

Conclusion: Cataract surgery without complication can widen the anterior chamber and lower the intraocular pressure, and can improve the visual acuity with short period of follow up, not like the traditional glaucoma surgery which needs long follow up and special care more than phacoemulsification.
\end{abstract}

Keywords: Uncomplicated Phacoemulsification, Intraocular Pressure.

\section{INTRODUCTION}

Cataract and glaucoma are the first and second leading causes of blindness worldwide. Although usually not severe enough to cause blindness, it is not surprising that these two diseases occur simultaneously in many patients. Many studies have demonstrated intraocular pressure reduction after cataract surgery. However, most data indicates that IOP reduction after cataract surgery is more significant and sustained than previously thought (Berdahl, 2009).

The magnitude of IOP reduction varies among patients. Factors which help to predict the amount of reduction could be beneficial for timing of surgical intervention. Previous studies have shown that shallower anterior chamber depth, shorter axial length and higher preoperative IOP are correlated with 
greater reduction in postoperative IOP (Zamani et al., 2011).

As the lens grows, the anterior lens capsule is displaced forward causing the zonules to place anteriorly directed traction on the ciliary body and uveal tract, which in turn compresses the canal of Schlemm and trabecular meshwork, as the ciliary body is displaced forward by the enlarging lens the tendons relax and the space between trabecular plates becomes narrowed. Phacoemulsification typically induces low grade inflammation in the immediate postoperative period. This may lower IOP by either decreasing aqueous production of ciliary body as seen in uveitis; or it could increase outflow similar to the mechanism of selective laser trabeculoplasty or prostaglandin analogues (Jamil et al., 2010).

One of the potential complications associated with increased vacuum is unacceptable anterior chamber instability. To maintain anterior chamber stability during surgery, more infusion is provided, resulting in transient elevations of intraocular pressure (IOP). Several studies have been conducted to assess the ocular damage due to the temporary high IOP reported that a $20-\mathrm{mmHg}$ increase in IOP for 5 minutes caused reduced blood flow to the optic nerve, retina, and choroid in healthy subjects. Acute IOP elevations for less than 1 minute may inhibit the retrograde transport of essential neurotrophins from the brain to the retina (Zhao et al., 2009).

The method of cataract extraction may influence the reduction of IOP. For example, there is a greater reduction in IOP after phacoemulsification compared to manual extra capsular cataract extraction (ECCE). The reduction in IOP lasts for at least 12 months after phacoemulsification in non-glaucomatous eyes (Pal et al., 2013).

The high cost of present methods for glaucoma screening is a barrier to the identification of people at high risk for glaucoma blindness. In essence, surgery has the potential to fulfill many features of an ideal approach to reduce intraocular pressure (IOP) compared with medications. It can lower the IOP to low teens, achieve long-term IOP reduction, minimize IOP fluctuations, lower the long term cost, and minimize systemic side effects (Venkatesh et al., 2009).

The aim of the work was to study and measure the effect of uncomplicated phacoemulsification on intraocular pressure using air puff machine and applanation tonometer at Alazhar University Hospitals.

\section{PATIENTS AND METHODS}

This was a prospective randomized study carried out on 40 eyes that underwent measuring IOP by air puff and applanation tonometer before phacoemulsification and IOL implantation and one day, one month, three months post-operative in $\mathrm{Al}$ Azhar University Hospitals.

An informed and written consent was taken from all the patients included in the study

\section{Inclusion criteria :}

1. Senile cataract.

2. Pre-operative clear cornea.

3. Uncomplicated phacoemulsification. 


\section{Exclusion criteria:}

1. Complicated and infantile cataract

2. Patients with previous intraocular surgeries.

3. Patients with corneal opacity.

4. Patients with intra operative complications.

5. Patients with ocular diseases affecting IOP.

\section{Pre-operative evaluation including}

1. Slit lamp examination to detect any segment abnormality.

2. Visual acuity and best corrected visual acuity.

3. IOP measuring using air puff and applanation tonometer.

4. Grading of cataract.

5. Grade of Van Herrick to determine anterior chamber angle.

\section{Operative technique:}

All surgeries were performed by the same surgeon. Following ocular anesthesia, an incision was made $3.2 \mathrm{~mm}$ into clear cornea. The anterior chamber was injected with a viscoelastic agent. Approximately 5 to $5.5 \mathrm{~mm}$ diameter continuous curvilinear capsulorhexis was performed. After the lens nucleus was (hydrodiscted), phacoemulsification (divide and conquer technique) was performed in the capsular bag. The lens cortex was sucked clean using an automatic irrigation/aspiration system. Before and after capsule polishing, the viscoelastic agent was injected into the capsular bag and an artificial lens was implanted into it. The viscoelastic agents were then sucked clean to restore the anterior chamber. All surgeries were successful and no complications occurred.

\section{Post-operative evaluation included}

1. Slit lamp evaluation of corneal clarity

2. Best corrected visual acuity.

3. IOP measurement using air puff and applanation tonometer one day, one month and three months post-operative.

4. Grading of Van Herrick to determine anterior chamber angle.

\section{Statistical Analysis:}

Data were collected, revised, coded and entered to the statistical package for social science (SPSS) version 23. The quantitative data were presented as mean, standard deviations and ranges. So, the pvalue was considered significant when $\mathrm{P}>$ 0.05 .

\section{RESULTS}

There was no statistically significant relation found between age and IOP measured by air puff tonometer at different times of measurement (table 1). 
Table (1): Comparison between Age <56 and Age >=56 regarding IOP measured by air-puff tonometer

\begin{tabular}{|c|c|c|c|c|c|}
\hline \multirow{2}{*}{\multicolumn{2}{|c|}{ IOP measured by air-puff tonometer }} & Age $<56$ & Age $>=56$ & \multirow{2}{*}{ Test value } & \multirow{2}{*}{ P-value } \\
\hline & & No. $=18$ & No. $=22$ & & \\
\hline \multirow{2}{*}{ Air puff before } & Mean \pm SD & $18.35 \pm 2.43$ & $19.50 \pm 3.28$ & \multirow{2}{*}{$-1.258 \bullet$} & \multirow{2}{*}{0.216} \\
\hline & Range & $15-25$ & $15-28$ & & \\
\hline \multirow{2}{*}{ AP after 1 day } & Mean \pm SD & $18.05 \pm 2.63$ & $19.45 \pm 3.17$ & \multirow{2}{*}{$-1.521 \bullet$} & \multirow{2}{*}{0.137} \\
\hline & Range & $15-22$ & $13-26$ & & \\
\hline \multirow{2}{*}{ AP after 1 week } & Mean \pm SD & $17.05 \pm 3.00$ & $17.80 \pm 2.75$ & \multirow{2}{*}{$-0.825 \bullet$} & \multirow{2}{*}{0.415} \\
\hline & Range & $13-23$ & $13-22$ & & \\
\hline \multirow{2}{*}{ AP after 1 month } & Mean \pm SD & $16.40 \pm 2.52$ & $17.80 \pm 3.17$ & \multirow{2}{*}{$-1.545 \bullet$} & \multirow{2}{*}{0.131} \\
\hline & Range & $13-21$ & $13-22$ & & \\
\hline \multirow{2}{*}{ AP after 3 months } & Mean \pm SD & $16.55 \pm 2.65$ & $17.70 \pm 3.18$ & \multirow{2}{*}{$-1.243 \bullet$} & \multirow{2}{*}{0.221} \\
\hline & Range & $12-22$ & $12-23$ & & \\
\hline \multirow{2}{*}{$\%$ change (1day) } & Mean \pm SD & $-1.21 \pm 11.16$ & $0.33 \pm 10.96$ & \multirow{2}{*}{$-0.528 \neq$} & \multirow{2}{*}{0.597} \\
\hline & Range & $-21.05-22.22$ & $-18.75-17.65$ & & \\
\hline \multirow{2}{*}{$\%$ change (1week) } & Mean \pm SD & $-1.21 \pm 11.16$ & $0.33 \pm 10.96$ & \multirow{2}{*}{$-0.528 \neq$} & \multirow{2}{*}{0.597} \\
\hline & Range & $-21.05-22.22$ & $-18.75-17.65$ & & \\
\hline \multirow{2}{*}{$\%$ change (1month) } & Mean \pm SD & $-10.42 \pm 9.66$ & $-8.25 \pm 11.96$ & \multirow{2}{*}{$-0.664 \neq$} & \multirow{2}{*}{0.507} \\
\hline & Range & $-26.32-16.67$ & $-25-17.65$ & & \\
\hline \multirow{2}{*}{$\%$ change (3months) } & Mean \pm SD & $-9.57 \pm 10.94$ & $-8.90 \pm 10.83$ & \multirow{2}{*}{$-0.406 \neq$} & \multirow{2}{*}{0.685} \\
\hline & Range & $-26.32-22.22$ & $-27.27-11.76$ & & \\
\hline
\end{tabular}

$\bullet:$ Independent t-test; $¥$ : Mann Whitney test

There was no statistically significant relation found between age and IOP measured by Goldmann applantation tonometer at different times of measurement (table 2).

Table (2) :Comparison between Age $<56$ and Age $\geq 56$ regarding IOP measured by Goldmann applantation tonometer

\begin{tabular}{|c|c|c|c|c|c|}
\hline \multirow{2}{*}{\multicolumn{2}{|c|}{$\begin{array}{c}\text { IOP measured by } \\
\text { Goldmann applanation tonometer }\end{array}$}} & Age $<56$ & Age $\geq 56$ & \multirow{2}{*}{ Test value } & \multirow{2}{*}{ P-value } \\
\hline & & No. $=18$ & No. $=22$ & & \\
\hline \multirow{2}{*}{ Before } & Mean \pm SD & $18.30 \pm 2.54$ & $19.45 \pm 3.41$ & \multirow{2}{*}{$-1.210 \bullet$} & \multirow{2}{*}{0.234} \\
\hline & Range & $15-26$ & $15-29$ & & \\
\hline \multirow{2}{*}{ After 1 day } & Mean \pm SD & $18.05 \pm 2.96$ & $19.10 \pm 3.16$ & \multirow{2}{*}{$-1.084 \bullet$} & \multirow{2}{*}{0.285} \\
\hline & Range & $15-24$ & $13-27$ & & \\
\hline \multirow{2}{*}{ After 1 week } & Mean \pm SD & $17.25 \pm 2.81$ & $17.85 \pm 2.54$ & \multirow{2}{*}{$-0.709 \bullet$} & \multirow{2}{*}{0.483} \\
\hline & Range & $14-23$ & $12-22$ & & \\
\hline \multirow{2}{*}{ After 1 month } & Mean \pm SD & $16.70 \pm 2.72$ & $17.90 \pm 2.81$ & \multirow{2}{*}{$-1.374 \bullet$} & \multirow{2}{*}{0.178} \\
\hline & Range & $12-22$ & $13-22$ & & \\
\hline \multirow{2}{*}{ After 3 months } & Mean \pm SD & $16.60 \pm 2.66$ & $17.80 \pm 2.97$ & \multirow{2}{*}{$-1.346 \bullet$} & \multirow{2}{*}{0.186} \\
\hline & Range & $12-22$ & $13-23$ & & \\
\hline \multirow{2}{*}{$\%$ change (1day) } & Mean \pm SD & $-1.03 \pm 12.31$ & $-0.97 \pm 12.57$ & \multirow{2}{*}{$-0.095 \ddagger$} & \multirow{2}{*}{0.924} \\
\hline & Range & $-21.05-21.05$ & $-23.53-18.75$ & & \\
\hline \multirow{2}{*}{$\%$ change (1week) } & Mean \pm SD & $-1.03 \pm 12.31$ & $-0.97 \pm 12.57$ & \multirow{2}{*}{$-0.095 \ddagger$} & \multirow{2}{*}{0.924} \\
\hline & Range & $-21.05-21.05$ & $-23.53-18.75$ & & \\
\hline \multirow{2}{*}{$\%$ change (1moth) } & Mean \pm SD & $-8.33 \pm 12.64$ & $-7.20 \pm 11.21$ & \multirow{2}{*}{$-0.666 \neq$} & \multirow{2}{*}{0.506} \\
\hline & Range & $-31.58-29.41$ & $-31.03-11.76$ & & \\
\hline \multirow{2}{*}{$\%$ change (3months) } & Mean \pm SD & $-8.94 \pm 12.16$ & $-7.78 \pm 11.73$ & \multirow{2}{*}{$-0.611 \neq$} & \multirow{2}{*}{0.541} \\
\hline & Range & $-26.32-29.41$ & $-27.59-17.65$ & & \\
\hline
\end{tabular}

•: Independent t-test; $\ddagger$ : Mann Whitney test 
There was no statistically significant relation found between age and anterior chamber angle before and after surgery (table 3).

Table (3): Comparison between Age $<56$ and Age $\geq 56$ regarding anterior chamber angle

\begin{tabular}{|c|c|c|c|c|c|}
\hline \multirow{2}{*}{\multicolumn{2}{|c|}{ Anterior chamber angle }} & Age $<56$ & Age $\geq 56$ & \multirow{2}{*}{ Test value } & \multirow{2}{*}{ P-value } \\
\hline & & No. $=18$ & No. $=22$ & & \\
\hline \multirow{2}{*}{ Before } & Mean \pm SD & $2.95 \pm 0.60$ & $2.90 \pm 0.79$ & \multirow{2}{*}{$0.225 \bullet$} & \multirow{2}{*}{0.823} \\
\hline & Range & $2-4$ & $2-4$ & & \\
\hline \multirow{2}{*}{ After } & Mean \pm SD & $3.85 \pm 0.37$ & $3.80 \pm 0.41$ & \multirow{2}{*}{$0.406 \bullet$} & \multirow{2}{*}{0.687} \\
\hline & Range & $3-4$ & $3-4$ & & \\
\hline \multirow{2}{*}{$\%$ change } & Mean \pm SD & $34.17 \pm 21.27$ & $39.17 \pm 35.98$ & \multirow{2}{*}{$-0.175 \ddagger$} & \multirow{2}{*}{0.861} \\
\hline & Range & $0-100$ & $0-100$ & & \\
\hline
\end{tabular}

•: Independent t-test; $\ddagger$ : Mann Whitney test

There was no statistically significant relation found between gender of the studied patients and IOP measured by Air puff tonometer at different times of measurement (table 4).

Table (4): Comparison between males and females regarding IOP measured by air puff tonometer

\begin{tabular}{|c|c|c|c|c|c|}
\hline \multirow{2}{*}{\multicolumn{2}{|c|}{$\begin{array}{c}\text { IOP measured by } \\
\text { air-puff tonometer }\end{array}$}} & Female & Male & \multirow{2}{*}{ Test value } & \multirow{2}{*}{ P-value } \\
\hline & & No. $=19$ & No. $=21$ & & \\
\hline \multirow{2}{*}{ Before } & Mean \pm SD & $19.16 \pm 3.55$ & $18.71 \pm 2.26$ & \multirow{2}{*}{$0.476 \bullet$} & \multirow{2}{*}{0.637} \\
\hline & Range & $15-28$ & $15-23$ & & \\
\hline \multirow{2}{*}{ After 1 day } & Mean \pm SD & $18.79 \pm 3.01$ & $18.71 \pm 2.99$ & \multirow{2}{*}{$0.079 \bullet$} & \multirow{2}{*}{0.937} \\
\hline & Range & $15-25$ & $13-26$ & & \\
\hline \multirow{2}{*}{ After 1 week } & Mean \pm SD & $17.53 \pm 3.10$ & $17.33 \pm 2.71$ & \multirow{2}{*}{$0.210 \bullet$} & \multirow{2}{*}{0.835} \\
\hline & Range & $13-23$ & $13-22$ & & \\
\hline \multirow{2}{*}{ After 1 month } & Mean \pm SD & $17.00 \pm 3.06$ & $17.19 \pm 2.86$ & \multirow{2}{*}{$-0.204 \bullet$} & \multirow{2}{*}{0.840} \\
\hline & Range & $13-22$ & $13-22$ & & \\
\hline \multirow{2}{*}{ After 3 months } & Mean \pm SD & $17.26 \pm 3.21$ & $17.00 \pm 2.76$ & \multirow{2}{*}{$0.279 \bullet$} & \multirow{2}{*}{0.782} \\
\hline & Range & $12-23$ & $12-22$ & & \\
\hline \multirow{2}{*}{$\%$ change (1day) } & Mean \pm SD & $-0.99 \pm 11.55$ & $0.06 \pm 10.64$ & \multirow{2}{*}{$-0.461 \neq$} & \multirow{2}{*}{0.645} \\
\hline & Range & $-20-22.22$ & $-21.05-13.33$ & & \\
\hline \multirow{2}{*}{$\%$ change (1week) } & Mean \pm SD & $-0.99 \pm 11.55$ & $0.06 \pm 10.64$ & \multirow{2}{*}{$-0.461 \neq$} & \multirow{2}{*}{0.645} \\
\hline & Range & $-20-22.22$ & $-21.05-13.33$ & & \\
\hline \multirow{2}{*}{$\%$ change (1moth) } & Mean \pm SD & $-10.77 \pm 10.00$ & $-8.04 \pm 11.54$ & \multirow{2}{*}{$-0.732 \neq$} & \multirow{2}{*}{0.464} \\
\hline & Range & $-25-17.65$ & $-26.32-16.67$ & & \\
\hline \multirow{2}{*}{$\%$ change (3months) } & Mean \pm SD & $-9.61 \pm 8.84$ & $-8.90 \pm 12.44$ & \multirow{2}{*}{$-0.041 \neq$} & \multirow{2}{*}{0.968} \\
\hline & Range & $-25-11.76$ & $-27.27-22.22$ & & \\
\hline
\end{tabular}

•: Independent t-test; + : Mann Whitney test

There was no statistically significant relation found between gender of the studied patients and IOP measured by
Goldmann applantation tonometer at different times of measurement (table 5). 
Table (5): Comparison between males and females regarding IOP measured by Goldmann applantation tonometer

\begin{tabular}{|c|c|c|c|c|c|}
\hline \multirow{2}{*}{\multicolumn{2}{|c|}{$\begin{array}{l}\text { IOP measured by Goldmann } \\
\text { applanation tonometer }\end{array}$}} & Female & Male & \multirow{2}{*}{ Test value } & \multirow{2}{*}{ P-value } \\
\hline & & No. $=19$ & No. $=21$ & & \\
\hline \multirow{2}{*}{ Before } & Mean \pm SD & $19.11 \pm 3.77$ & $18.67 \pm 2.22$ & \multirow{2}{*}{$0.454 \bullet$} & \multirow{2}{*}{0.653} \\
\hline & Range & $15-29$ & $15-24$ & & \\
\hline \multirow{2}{*}{ After 1 day } & Mean \pm SD & $18.42 \pm 2.87$ & $18.71 \pm 3.30$ & \multirow{2}{*}{$-0.298 \bullet$} & \multirow{2}{*}{0.767} \\
\hline & \begin{tabular}{|l|} 
Range \\
\end{tabular} & $15-23$ & $13-27$ & & \\
\hline \multirow{2}{*}{ After 1 week } & Mean \pm SD & $17.74 \pm 2.68$ & $17.38 \pm 2.69$ & \multirow{2}{*}{$0.418 \bullet$} & \multirow{2}{*}{0.678} \\
\hline & Range & $14-23$ & $12-22$ & & \\
\hline \multirow{2}{*}{ After 1 month } & Mean \pm SD & $17.16 \pm 2.95$ & $17.43 \pm 2.71$ & \multirow{2}{*}{$-0.302 \bullet$} & \multirow{2}{*}{0.764} \\
\hline & Range & $12-22$ & $13-22$ & & \\
\hline \multirow{2}{*}{ After 3 months } & Mean \pm SD & $17.37 \pm 3.24$ & $17.05 \pm 2.52$ & \multirow{2}{*}{$0.352 \bullet$} & \multirow{2}{*}{0.727} \\
\hline & Range & $12-23$ & $13-22$ & & \\
\hline \multirow{2}{*}{$\%$ change (1day) } & Mean \pm SD & $-2.44 \pm 11.57$ & $0.30 \pm 13.02$ & \multirow{2}{*}{$-0.651 \neq$} & \multirow{2}{*}{0.515} \\
\hline & Range & $-20.69-21.05$ & $-23.53-18.75$ & & \\
\hline \multirow{2}{*}{$\%$ change (1week) } & Mean \pm SD & $-2.44 \pm 11.57$ & $0.30 \pm 13.02$ & \multirow{2}{*}{$-0.651 \neq$} & \multirow{2}{*}{0.515} \\
\hline & Range & $-20.69-21.05$ & $-23.53-18.75$ & & \\
\hline \multirow{2}{*}{$\%$ change (1moth) } & Mean \pm SD & $-9.44 \pm 10.05$ & $-6.25 \pm 13.26$ & \multirow{2}{*}{$-0.748 \neq$} & \multirow{2}{*}{0.454} \\
\hline & Range & $-31.03-11.76$ & $-31.58-29.41$ & & \\
\hline \multirow{2}{*}{$\%$ change ( 3 months) } & Mean \pm SD & $-8.48 \pm 11.22$ & $-8.25 \pm 12.59$ & \multirow{2}{*}{$-0.041 \neq$} & \multirow{2}{*}{0.967} \\
\hline & Range & $-27.59-17.65$ & $-26.32-29.41$ & & \\
\hline
\end{tabular}

$\bullet:$ Independent t-test; $;$ : Mann Whitney test

There was no statistically significant relation found between gender of the studied patients and anterior chamber angle before and after surgery (table 6).

Table (6): Comparison between males and females regarding anterior chamber angle

\begin{tabular}{|c|c|c|c|c|c|}
\hline \multirow{2}{*}{\multicolumn{2}{|c|}{ Anterior chamber angle }} & Female & Male & \multirow{2}{*}{ Test value } & \multirow{2}{*}{ P-value } \\
\hline & & No. $=19$ & No. $=21$ & & \\
\hline \multirow{2}{*}{ Before } & Mean \pm SD & $2.84 \pm 0.60$ & $3.00 \pm 0.77$ & \multirow{2}{*}{$-0.714 \bullet$} & \multirow{2}{*}{0.479} \\
\hline & Range & $2-4$ & $2-4$ & & \\
\hline \multirow{2}{*}{ After } & Mean \pm SD & $3.79 \pm 0.42$ & $3.86 \pm 0.36$ & \multirow{2}{*}{$-0.550 \bullet$} & \multirow{2}{*}{0.585} \\
\hline & \begin{tabular}{|l|} 
Range \\
\end{tabular} & $3-4$ & $3-4$ & & \\
\hline \multirow{2}{*}{$\%$ change } & Mean \pm SD & $36.84 \pm 20.47$ & $36.51 \pm 35.99$ & \multirow{2}{*}{$-0.658 \neq$} & \multirow{2}{*}{0.511} \\
\hline & Range & $0-100$ & $0-100$ & & \\
\hline
\end{tabular}

$\bullet:$ Independent t-test; + : Mann Whitney test

\section{DISCUSSION}

As the lens grows, the anterior lens capsule is displaced forward causing the zonules to place anteriorly directed traction on the ciliary body and uveal tract, which in turn compresses the canal of Schlemm and trabecular meshwork, as the ciliary body is displaced forward by the enlarging lens the tendons relax and the space between trabecular plates becomes narrowed. Phacoemulsification typically induces low grade inflammation in the immediate postoperative period. This may lower IOP by either decreasing aqueous production of ciliary body as seen in uveitis; or it could increase outflow similar to the mechanism of selective laser trabeculoplasty or prostaglandin analogues (Jamil et al., 2010). 
Surgeons have traditionally felt that cataract surgery lowers IOP in open angle glaucoma (OAG) only slightly and temporarily - despite a paucity of robust data. In contrast, current data demonstrates a greater and more sustained IOP reduction. As such, cataract surgery may be a safe alternative to glaucoma surgery in some patients and could shift the surgeon's approach in treatment of concurrent cataract and glaucoma (Berdahl, 2009).

The current study was designed to compare the intraocular pressure before and after uncomplicated phacoemulsification surgery. In our study, there was a statistically significant difference between IOP before and after phaco.

A supporter study done by Baek et al. (2019) in total, 754 eyes of 754 patients, specifically 106 patients with glaucoma and 648 patients with no glaucoma (i.e., healthy subjects) were enrolled. The phacoemulsification effected a reduction of IOP: $1.03 \pm 3.72 \mathrm{mmHg}$ in healthy subjects and $1.08 \pm 3.79 \mathrm{mmHg}$ in glaucoma patients at postoperative 1 year. There were negative coefficients of IOP until 1 year of follow up, but the IOP change gradually showed a less steeply decreasing slope (correlation coefficient: 0.993), compared with those for 1 week and 1 month of follow up (correlation coefficients: -1.893 and -1.540 , respectively). In the multivariate analysis, age and preoperative IOP showed significant associations with postoperative IOP change.

Majstruk et al. (2019) agreed with our study as they found that one year after phacoemulsification, IOP decreased by a mean $1.15 \pm 3 \mathrm{mmHg}(6.8 \pm 18.1 \%)$ and the number of glaucoma medications remained unchanged with a difference of $0.1 \pm 0.43$. Higher preoperative IOP was associated with a greater IOP decrease after 1 year of follow-up. One and 7 days after cataract surgery, 12.9 and $4.2 \%$ of the eyes had IOP spikes $>30 \mathrm{mmHg}$, respectively. One year after cataract surgery, $75.7 \%$ of the POAG eyes maintained the same number of glaucoma medications, while $17.1 \%$ had a decrease and $7.2 \%$ of the eyes required adding glaucoma medications.

The magnitude of IOP reduction varies among patients. Factors which help to predict the amount of reduction could be beneficial for timing of surgical intervention. Previous studies have shown that shallower anterior chamber depth, shorter axial length and higher preoperative IOP are correlated with greater reduction in postoperative IOP (Zamani et al., 2011).

Similar results found by Picoto et al. (2014) who examined 51 eyes without glaucoma and 50 eyes with glaucoma. They found the medium IOP was $16.08 \pm$ 3.04 before surgery, $14.69 \pm 2.7$ after 12 months and $14.21 \pm 3.56$ at 24 months. The medium value of IOP reduction before surgery to 12 months was $1.49 \pm$ 2.98. IOP measured before surgery differed statistically from IOP at 12 and 24 months. They found that cataract surgery with IOL implantation reduced IOP by $1.48 \pm 2.98 \mathrm{mmHg}$, on average. The decrease was sustained after 24 and 36 months both in glaucoma and nonglaucoma eyes.

Another proponent study done by Mansberger et al. (2013) confirmed that 
cataract surgery with intraocular lens implantation lowers IOP. Cataract surgery decreased postoperative IOP by $4.0 \mathrm{~mm}$ $\mathrm{Hg}$, resulting in a $16.5 \%$ decrease from preoperative IOP, which was sustained at that level for at least a year. The effect persisted, but diminished over the next 2 years. The group with the highest preoperative IOP had the largest percentage drop in postoperative IOP.

On the other hand, Muge et al. (2013) made a study on 812 eyes of 584 patients who underwent uncomplicated phacoemulsification were evaluated. There were 330 men and 254 women ranging between the age of 26 and 89 years $(65.4 \pm 9.8$ years $)$. The preoperative, postoperative first day (day 1), first week (day 7), and first month (day 30) IOP values were analyzed. The mean IOP was $15.6 \pm 4.3 \mathrm{mmHg}$ preoperatively. Postoperatively, that was changed to 19.7 $\pm 9.0 \mathrm{mmHg}$ at day $1,12.7 \pm 4.5 \mathrm{mmHg}$ at day 7 , and $12.8 \pm 3.7 \mathrm{mmHg}$ at day 30 . The factors such as surgeon, presence of PXF, diabetes, surgical incision site, and trypan blue were not related to the postoperative high IOP. The only factor that related to high IOP at all visits was glaucoma.

Gokhale and Patterson (2009) reported a review of 630 cases of cataract extraction with lens implant concluded that elevated IOP was a transient and of benign occurrence. In nearly all patients, pressures returned to baseline with or without treatment. The causes of the elevated IOP are likely multifactorial. Major factors include preexisting compromise of outflow facility and retained ophthalmic viscosurgical devices (OVDs). Surgical trauma, watertight wound closure, retained lenticular debris, the release of iris pigment, hyphema, and inflammation are also thought to contribute to elevations in IOP. The skillfulness of the surgeon has been implicated as well. Increased surgical experience is correlated with a decreased risk for ocular hypertension following cataract extraction.

A study done by Armstrong et al. (2017) found that a $12 \%, 14 \%, 15 \%$, and $9 \%$ reduction in IOP from baseline occurred 6, 12, 24, and 36 months respectively after phacoemulsification.

\section{CONCLUSION}

Cataract surgery without complication can widen the anterior chamber and lower the intraocular pressure and can improve the visual acuity with short period of follow up not like the traditional glaucoma surgery which need long follow up and special care more than phacoemulsification.

\section{REFERENCES}

1. Armstrong $\mathbf{J}$, Wasiuta $\mathrm{T}$, Kiatos E, Malvankar M and Hutnik C (2017): The Effects of Phacoemulsification on Intraocular Pressure and Topical Medication Use in Patients with Glaucoma: A Systematic Review and Meta-analysis of 3-Year Data. J Glaucoma, 26(6):511-22.

2. Baek SU, Kwon S, Park IW and Suh W (2019): Effect of Phacoemulsification on Intraocular Pressure in Healthy Subjects and Glaucoma Patients. J Korean Med Sci., 34(6):47-49.

3. Berdahl PJ (2009): Cataract surgery to lower intraocular pressure. Mid East Afr J Ophthalmol., 16:119-22.

4. Gokhale PA and Patterson E (2009): Elevated Intraocular Pressure After Cataract Surgery. Cataract Surgery in the Glaucoma 
Patient, pbl Springe, 1st ed., New York, 5:515 .

5. Jamil AZ, Iqbal $Z$, Rahman $Z U$ and Khurram AM (2011): Effect of Phacoemulsification on Intraocular Pressure Journal of the College of Physicians and Surgeons Pakistan. 21(6):347-50.

6. Majstruk L, Leray B, Bouillot A, Michée S, Sultan G, Baudouin $C$ and Labbé A (2019): Long term effect of phacoemulsification on intraocular pressure in patients with medically controlled primary open-angle glaucoma. BMC Ophthalmol, 19: 149.

7. Mansberger SL, Gordon MO, Jampel H, Bhorade A, Brandt JD, Wilson B and Kass MA (2013): Reduction in intraocular pressure after cataract extraction. The Ocular Hypertension Treatment Study, 119(9):182631 .

8. Muge CK, Selcuk S, Rana AY, Handan C and Ayden AY (2013): Risk factors for intraocular pressure rise following phacoemulsification. Indian J Ophthalmol., 61(3):115-8.

9. Pal VK, Agrawal A, Suman S and Pratap VB (2013): Long-Term Change in Intraocular Pressure after Extracapsular Cataract Extraction with Posterior Chamber Intraocular Lens Implantation Versus
Phacoemulsification with Posterior Chamber Intraocular Lens Implantation in Indians. Mid East Afr J Ophthalmol., 20(4):332-5.

10. Picoto M, Galveia J, Almeida A, Patr?cio S, Spohr H, Vieira $P$ and Vaz F (2014): Intraocular pressure (IOP) after cataract extraction surgery. Revista Brasileira de Oftalmologia, 73(10): 0034-7280.

11. Pohjalainen T, Vesti E, Uusitalo RJ, Laatikainen L (2001): Phacoemulsification and intraocular lens implantation in eyes with open-angle glaucoma. Acta Ophthalmologica Scandinavica, 79(3):313-6.

12. Venkatesh R, Ramakrishnan R, Krishnadas R, Sathyan $P$ and Robin AL (2009): Managing Cataract and Glaucoma in the Developing World - Manual Small Incision Cataract Surgery (MSICS) Combined with Trabeculectomy. Cataract Surgery in the Glaucoma Patient, 73-82.

13. Zamani M, Feghhi $M$ and Azarkish A (2011): Early Changes in Intraocular pressure following phacoemulsification, J Ophthalmic Vis Res., 8(1):25-31.

14. Zhao Y, Li X, Tao A, Wang $J$ and Lu F (2009): Intraocular Pressure and Calculated Diastolic Ocular Perfusion Pressure during Three Simulated Steps of Phacoemulsification In Vivo, Invest Ophthalmol Vis Sci., 50: 2927-31. 


\section{تأثير إزالة المياه البيضناء بالموجات فوق الصوتية الفير مصحوب بمضاعمات علي ضغط المين}

السيا مصطفي عليوة- حسن متولي بيومي - عاطف سمير حامد محمد قسم طب وجراحة العيون- كلية الطب- جامعة الأزهر - القاهرة

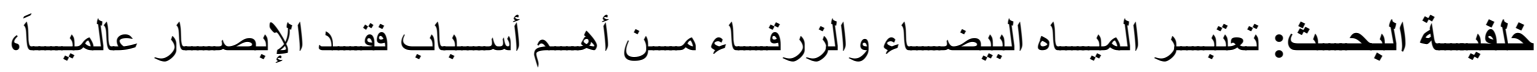

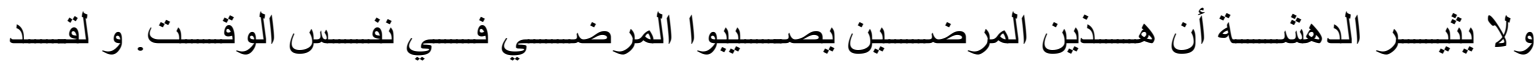

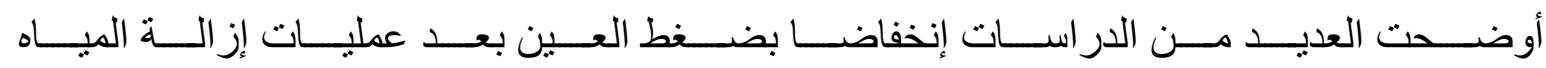

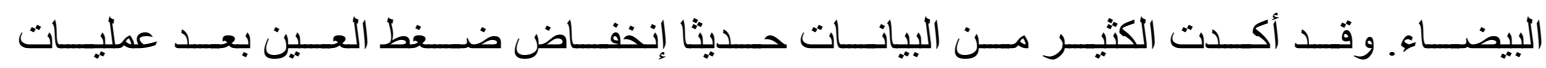
إز الة المياه البيضاء بصورة واضحة ومستمرة أكثر مما أعتقدنا من قبل.

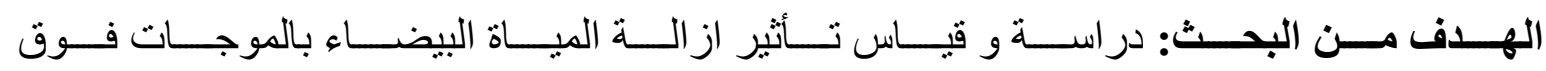
الصـــوتية الغيــر مصــحوب بمضـــاعفات ومــع زر اعــة عدســة داخـلـل العـين عـن طريــق

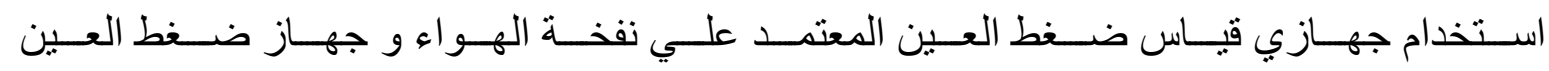
المعتمد علي مقياس تسطح القرنية و ذلك في مستشفيات جامعة الأزهر.

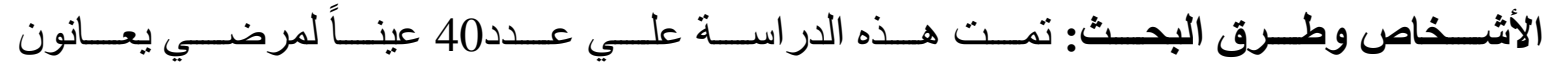

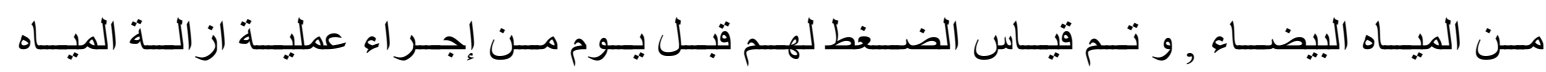

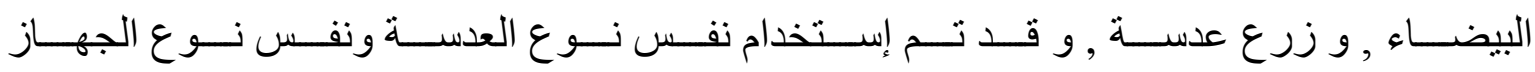

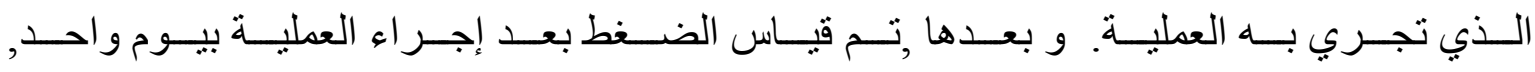
ثم اسبوع ,ثم شهر رثم ثلاثة أشهر.

النتائج: انخفض ضغط العين بعد إز الة المياه البيضاء بصورة واضحة.

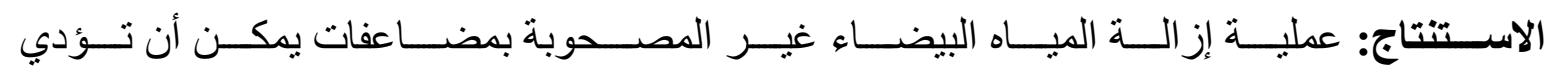

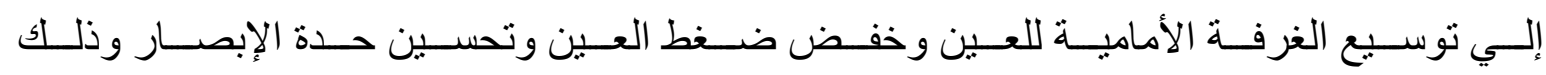

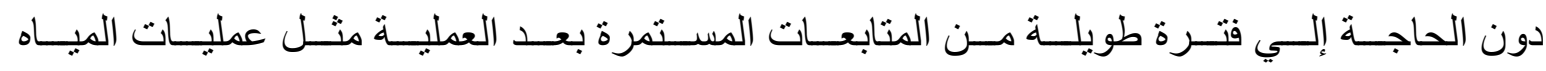
الزرقاء التقليدية والتي تحتاج إلي منابعات أكثر و عناية خاصة. 